\title{
Erratum
}

Frederik Stork · Marc Uetz

\section{On the generation of circuits and minimal forbidden sets}

\section{Math. Program, Ser. A, http://dx.doi.org/10.1007/s10107-004-0512-0}

Received: October 22, 2002 / Accepted: February 10, 2004

Published online: 31 May 2004 - (C) Springer-Verlag 2004

In Section 4.2 of the paper, the labels of the diagrams in Figure 3 are misprinted. The ordinate labels must be "average $|F|$ " instead of "average $|\mathcal{F}|$ " for all three diagrams. These diagrams display the average cardinality $|F|$ of the minimal forbidden sets, and not the average number $|\mathcal{F}|$ of minimal forbidden sets.

F. Stork: ILOG Deutschland GmbH, Ober-Eschbacher Straße 109, D-61352 Bad Homburg, Germany. e-mail: fstork@ilog.de

M. Uetz: Universiteit Maastricht, Faculty of Economics and Business Administration, Quantitative Economics, P.O. Box 616, NL-6200 MD Maastricht, The Netherlands.

e-mail: m.uetz@ke.unimaas.nl 